\title{
Research on Cross-Border Marketing and Traditional Brand Image Remodeling__ Take the Old Brand "White Rabbit" as an Example
}

\author{
Xianglin $\mathrm{He}^{1}$ \\ ${ }^{1}$ The College of Literature and Journalism of Sichuan University, China \\ Correspondence: Xianglin He, Sichuan University, Chengdu 610207, China.
}

Received: April 10, 2020

Accepted: April 29, 2020

Available online: May 8, 2020

doi:10.11114/aef.v7i4.4846

URL: https://doi.org/10.11114/aef.v7i4.4846

\begin{abstract}
"White Rabbit" toffee is a traditional Chinese brand with high reputation that bears the good memories of Chinese people. Facing fierce market competition, "White Rabbit" shares user groups with other related or complementary brands through cross-border marketing. Meet the diverse and personalized needs of consumers; use fashion, art, entertainment and other elements to enrich the brand 's connotation, and increase brand influence and added value; while innovating and changing, finely control the quality and rhythm of brand cross-border marketing and always maintain their brand identity and brand advantage. Through these measures, the "White Rabbit" old-fashioned brand calmly crossed the development bottleneck and gained new life, and the development path explored has brought a lot of inspiration to other old-fashioned brands and brand cross-border marketing strategies.
\end{abstract}

Keywords: traditional brands, cross-border marketing, rebranding

\section{Introduction}

\subsection{The Issue of "Cross-Border Marketing and Traditional Brand Image Remodeling"}

With the rapid economic development and the accelerated transformation of society, the market environment is undergoing rapid changes. In the new era of consumption, it is becoming increasingly important to win consumer attention and recognition while paying attention to the product or brand advantages. Oriented marketing has become a new trend in the market. Today, with the increasing popularity of the Internet, marketing methods have also been constantly updated, ushering in a new wave of "cross-border" marketing. "Cross-border" has become a business trend, bringing brand communication effects that cannot be underestimated, and is the most fashionable marketing model at present. Cross-border marketing refers to the brand's independent or combined with other brands in the non-brand industry. Based on the same or similar consumer groups and consistent goals, learn from each other's strengths and leverage their advantages to launch products that are not the main business of the brand in order to grasp consumer psychology. To meet the needs of consumers, and finally reach the goal of "1 $+1>2$ ". Some brands have strong complementarity and relevance, and the consumer groups are highly overlapped, and they can easily achieve each other to achieve a win-win situation; some brands seem to have nothing to do with each other. There are similarities in appeals. Cross-border is an infiltration and fusion between two brands. Establishing a cross-border relationship is not blindly following the trend, but based on an objective evaluation of its own characteristics. It carefully finds common points with other brands and injects its own brand personality. Cooperate with other brands to expand communication channels, integrate brand resources, and give brands a sense of freshness. Compared with traditional marketing communication, cross-border marketing is a new marketing communication thinking. In today's environment, brand cross-border marketing must grasp three core concepts. First, we must focus on consumer demand to realize brand value; second, create creative products and increase effective contact points; finally, customize reasonable plans, integrate brand resources, and expand communication channels.

Based on the analysis of cross-border marketing function, this paper attempts to build a relationship model between cross-border marketing and value creation in the mobile Internet environment. Through the cooperation between brands, the designed content will be delivered to customers in the form of affiliate marketing to provide information and entertainment for customers, and at the same time, the relationship between customers will be closer, so as to realize customer value and enterprise value. In this way, we can create the unique personalized experience of customers through interaction, and realize the value creation of all parties involved in the interaction. Through cross-border can 
constantly create new value, it can be seen that cross-border marketing is indeed an important way to achieve brand image reconstruction.

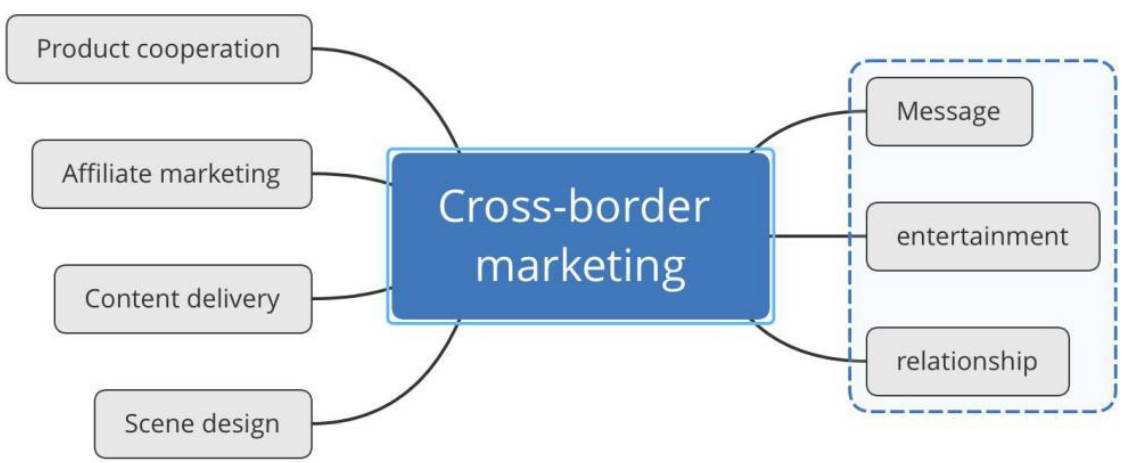

"Time-honored" brands are often a symbol of quality. They are unique, have a distinct traditional background, and their brand image is often deeply rooted. In the face of increasingly fierce market competition, the development of some "old-famous" brands has entered a bottleneck period, and how to rejuvenate it has become an urgent issue. If the economy and culture in the new environment cannot be adapted in time, the old brands will decline and even be eliminated by the market. In 2017, in the "Guiding Opinions on Promoting the Reform and Development of Time-honored Brands" jointly issued by the 16 departments including the Ministry of Commerce, it was explicitly mentioned that "to promote the time-honored brands to adapt to new changes in consumer demand and the Internet + New trends ... promote the heritage and innovation of time-honored brands and improve market competitiveness. " The biggest dilemma facing traditional Chinese brands is that the old generation of loyal consumers is getting old, the products have lost their applicability to them, and the brand lacks recognition among young consumers. In order to achieve brand rejuvenation, domestic brands should seize the opportunity of the current national boom and innovate marketing models. In recent years, traditional domestic brands, led by "White Rabbits", have paid more and more attention to rebranding and attracted more new consumer groups. "White Rabbit" actively meets consumer demand, and through cross-border marketing methods, it arouses the attention and discussion of consumers, enabling them to re-understand and understand traditional brands and achieve new life. The cross-border flash event almost allowed "White Rabbit" to sweep across major social platforms overnight, becoming a tide brand of online red national goods, and brought considerable sales and traffic to "White Rabbit". However, the "White Rabbit" has not been changed by the sudden red restrictions, but has concentrated on the old brand "high quality" tradition. The cross-border aspect is also comprehensive and efficient, maintaining the long-established development foundation through continuous daily operations, and controlling the pace of cross-border marketing.

"White Rabbit" was born in 1959, and has accompanied countless people through their childhood. It is the childhood memory of generations. As a time-honored brand, "White Rabbit" is the originator of the toffee industry and has a very high reputation at home and abroad. Newly married couples in the past must have a White Rabbit toffee in their wedding candy; every New Year's holiday, the family will buy a pack of White Rabbit toffee for children; the "White Rabbit" also created a "milk "Sugar diplomacy." It is said that Kissinger took away the White Rabbit toffee after his visit. Nowadays, under the background of the prevailing trend of domestic products, White Rabbit has grasped and continued its own brand cultural heritage, and seeks change through cross-borders, becoming a young and cool brand that can bear the time polishing. How to better grasp the current market, which has a special interest in vintage, traditional culture and national customs, better integrate the trendy lifestyle with traditional culture, and attract more affectionate consumers to complete the remodeling of their own image The rabbit undoubtedly brought some inspiration.

The author collected the Baidu index keyword search data of Baidu Index, the world 's largest Chinese search engine and the largest Chinese website, and obtained the following Baidu index keyword search trend chart. In general, from 2012 to 2015, the fluctuations were relatively stable, with two large fluctuations in 2016; after that, they continued to stabilize for a period of time, and in 2019, they experienced large fluctuations.

The reason for the peak of point A is that in 2015, a domestic enterprise launched a cottage version of a large white rabbit popsicle, and the "White Rabbit" said it would defend its rights. The appearance of the cottage version shows the little value of the "White Rabbit" cross-border, which is also an important reason why the "White Rabbit" chose to embark on the cross-border road. The occurrence of the peaks at points $\mathrm{B}$ and $\mathrm{C}$ is closely related to the information that 
the value of the white rabbit for French packaging has increased by nine times. At this time, it was the first time that the white rabbit tried to launch a French gift box with the international big brand Agnes.b. It can be seen that this cross-border has caused an upsurge in the media and society. The two peak points D and E that appeared in 2019 were also caused by White Rabbit's attempt to cross the border with Mejiajing to launch White Rabbit lipstick and genuine White Rabbit net red ice cream.

From the trend chart, it can be seen that the search trend of "White Rabbit" was relatively stable before the attempt to cross the border, and its brand popularity was relatively flat. After continuously trying to cross the border, it brought a lot of popularity to the brand and reached a good promotion effect. Although the enthusiasm is temporary and transient, being able to be effectively exposed to the public view is also very helpful for old brands.

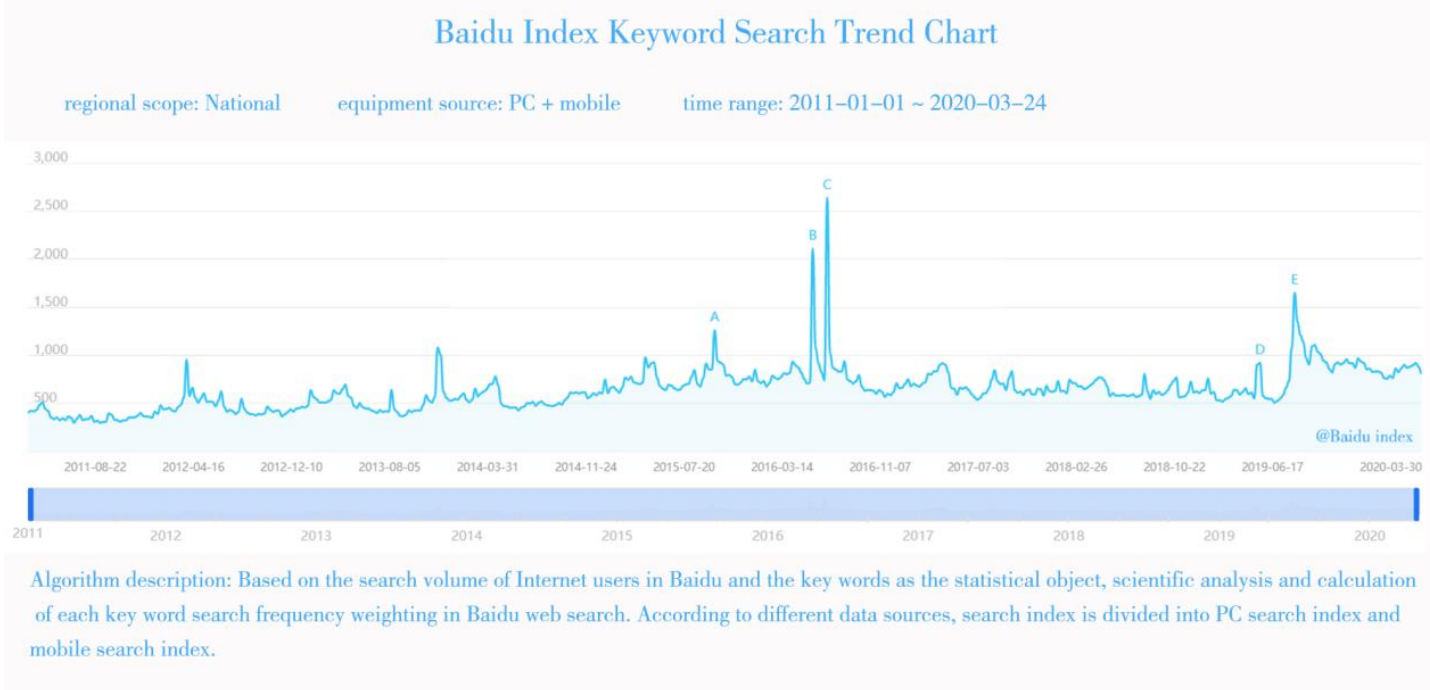

Chart 1. Baidu Index Keyword Search Trend 2011-2020

This article will start with the cross-border marketing of different types of White Rabbit brands, and analyze how brands can achieve their image change through efficient and comprehensive cross-border marketing.

\subsection{Literature Review}

The research results on cross-border marketing and traditional brand image remodeling can be roughly divided into two types: periodicals and research papers. Relevant domestic research started in the stage of rapid economic development in China. The author conducted an advanced search in the CNKI database under the title of "cross-border marketing", and obtained a total of 486 academic papers with a time span from 2006 to 2020; "Cross-border" is an advanced search for the title, and a total of 215 academic papers have been obtained with a time span from 1989 to 2020 . There are both concentrated debate articles and scattered papers, and most of them are scattered in research topics such as cross-border marketing and the new consumer era, cross-border marketing and brand marketing communication, Internet + and digital communication.

Foreign research on cross-border marketing has undergone considerable development for more than two decades. It can be traced back to 1999. When American brand Puma and clothing designer Jil Sander jointly launched high-end shoes, CEO Jochen Zeitz put forward the concept of "cross-border cooperation", and subsequently, other companies have also poured into the wave of cross-border. Research on brand image formation has also been going on for a long time. Since Ogway put forward the concept of brand image, most well-known scholars have expressed the theoretical ideas related to brand image in their writings. Nowadays, the research and knowledge of related theories are continuously enriched and deepened.

\subsection{Research Methods}

This article mainly uses case study method, text analysis method and literature method for analysis. Taking the well-known domestic long-established confectionery brand — White Rabbit as the research object, it selects its more successful cross-border marketing communication case for research and analysis. As a traditional brand, White Rabbit has tried hard in various cross-borders in recent years, hoping to get rid of the "old" label through different forms of 
cross-border expressions, and make traditional brands re-enter the public's horizon. In the process, we did not forget to link the essence of our traditional brand with the traditional culture, further establish the brand personality, create the distinctive characteristics of the brand, and finally achieve the purpose of rebranding the brand. Nowadays, looking at the "White Rabbit" several cross-border crossings since 2016, they are all relatively successful, so it is also very valuable for other similar brands.

The Chinese literature of this article mainly comes from the collection of relevant research literature on CNKI, the official Weibo of "White Rabbit", and the content on the "White Rabbit" WeChat public platform. It is organized, classified, summarized, and reanalyse based on the collected literature. Collect the entire historical development process of "White Rabbit" brand construction, and conduct carding and research; explore the relationship between cross-border research and brand image.

\section{Brand and Brand Cross-Border: Sharing User Groups and Meeting New Needs}

Generally, the products of traditional brands have only a single characteristic, and both the consumer group and the demand for the brand are relatively thin. Different brands can start from the original field, and then use the elements of creativity, fashion, art and other elements to seek new areas for marketing and communication based on the traditional field. In this process, different brands can not only consolidate the common consumer group, but also bring the non-crossed consumer groups closer to the cross, allowing irrelevant consumers to enter the common field to generate new demand. Based on the traditional characteristics of the brand, cross-border marketing can maximize the integration of brand resources, break through traditional marketing thinking, gain insight into new consumer needs, and then gain the favor of consumers in this new environment.

Therefore, in order to expand the "White Rabbit" consumer's demand area to be more diversified and meet the new requirements of creativity and fashion, a cross-border model of brands in different fields can be adopted, and the force of the "White Rabbit" brand can be extended with brand complementarity. Candy and clothing, candy and fragrance, candy and beverage seem to belong to different fields, but they have unique and delicate connections. Candy and clothing are common items in people's lives. Of course, the demand for clothing by consumers of traditional and old-fashioned sweets such as "White Rabbits" is certainly not simple, including factors such as nostalgia and fashion. Candies and fragrances can stimulate people's taste. The taste of "White Rabbit" is sweet, childhood taste, and perfume can give consumers stimulating odor. When perfume encounters "White Rabbit", consumers do not need to try the real thing. It seems that you can smell the perfume of White Rabbit. This synaesthesia can shorten the distance between the product and the consumer, and constantly "brush the presence" near the consumer, so that the two products are rooted in the consumer's life. At the same time, perfume and toffee are the products that young people are passionate about. Among them, there are nostalgic "toffee control" and there is a "perfume control" in pursuit of quality life. The collaboration of the two products expand the crossed groups and make irrelevant groups enter the common areas. Candy and beverages are snacks that young consumers like to eat. However, simple candy implanted ads can hardly give consumers a delicious and sweet pleasure. The organic combination of confectionery and beverage pop-up stores and online and offline cross-border marketing can allow consumers to be immersive and experience the sweetness of candy blended into beverages, suitable for young people seeking freshness. The cooperation between brands can enrich the connotation of the brand and expand the business field. It can also strengthen and consolidate existing consumer groups and expand new consumer groups by spanning the framework of traditional brands, shaping a brand new brand image and creating a more intense brand association.

Maxam is a famous Chinese skin care brand. "White Rabbit" and Maxam are both long-established traditional domestic brands, both from Shanghai. They both have an indelible impression in the minds of Chinese consumers and are seeking new breakthroughs. In the era of lack of material, both can be regarded as the leading brands in their respective fields, Shanghai Light, China's star products. In 2018, the 59-year-old "White Rabbit" and the 56-year-old Maxam jointly launched a White Rabbit toffee-flavored lipstick, which can be described as a strong partnership: one is that both are old-fashioned domestic products, and can emotionally undertake existing consumption nostalgic feelings of the consumer group; secondly, at the product level, the sweetness of both taste and smell can be combined to stimulate consumers' senses and bring a richer consumer experience to consumers; thirdly, lipstick continues the classic red and blue packaging of white rabbit milk sugar, has high visual recognition and can resonate with consumers. Finally, as a dairy product, the candy is soft and sweet on the tip of the tongue. The cream is also a creamy and nourishing lotion texture, both of which have great affinity. When two old brands merge in unexpected and reasonable ways, it's amazing and topical. On the day of product launch, the first batch of 920 "White Rabbit" lipsticks were snatched out within 30 seconds of being launched. When consumers use this product, conquered by its cuteness and classic. The use value of the product is also reflected, and the milky fragrance and moisturizing feeling are accepted and loved in a pleasant and comfortable mood. There is no doubt that this cross-border expansion has opened up the "White Rabbit" product category, making this toffee brand closer to the lives of consumers, more youthful and fashionable, and allowing the old 
brands to achieve the extension and expansion of the original image .

The brand's cross-border marketing focuses on grasping the growing consumer demand of consumers and satisfying consumer demand with outstanding products. As a long-established confectionery brand, "White Rabbit" is exploring the secret of long-lasting prosperity. It is very clever in cross-border brand selection. In addition to cooperating with traditional incumbents, it also tries to find other ways to develop creative categories. Cross-border cooperation between the established brand and the new wave brand is a symbiotic relationship. The target group and the brand concept are consistent to a certain extent. The old brand can help the new brand to increase its popularity. The new brand can in turn promote the transformation and upgrade of the old brand. In May 2019, "White Rabbit" and Smell Library cooperated to launch products such as White Rabbit Toffee Eau De Toilette, Fragrance Moisturizing Bath Milk, Fragrance Moisturizing Body Milk, Fragrance Moisturizing Hand Cream and Car Aromatherapy. The younger generation who likes to hunt for novelties, early adopters, and preference for domestic products is a consumer group shared by the two brands. In response to the actual needs of target consumers, the goal of "White Rabbit" is to turn it into a brand that young people are willing to share. In the end, the product incorporates elements such as the rabbit's image of the Great White Rabbit brand, red, blue and black classic colors, with a retro and highly recognizable image that makes the perfume unscented and smells first. Just after zero on the day of product launch, four thousand sets were sold in an instant, hand cream and perfume sold over 15,000, and 610 sets of 610 yuan White Rabbit tide packs specially prepared for Children's Day were sold out in three seconds; \# The rabbit perfume\# has read 330 million on Weibo. In this cross-border cooperation, the two brands can be described as having similar fragrances, and both sides have benefited greatly. Together, they have enhanced the brand's competitiveness and brand added value. The Smell Library has enhanced its brand awareness with the help of the "White Rabbit" brand name; "It has attracted the attention of the younger generation of consumers, shed the brand's outdated and old-fashioned outerwear, and helped the brand spread and extend in the new environment.

\section{Brand Cross-Border by Borrowing Elements: Enrich Brand Connotation and Increase Brand Touch Points}

Brands rely on other elements to achieve cross-borders, which can effectively enrich the connotation of the brand and increase the opportunity for the brand to engage with consumers. In order to achieve the best effect of borrowing elements across borders, the attributes and personality of the brand itself should be considered, and appropriate elements should be selected. Successful cross-border use of elements can bring additional returns to the brand and enhance brand competitiveness. With the help of different elements, you can achieve different communication effects and bring different benefits to the brand. Based on the attributes of the White Rabbit's toffee, the "White Rabbit" tried to integrate various elements, actively transformed, and achieved remarkable success. The following will briefly discuss the case of borrowing elements of the White Rabbit.

\subsection{Brand Cross-Border Fashion: Expanding Brand Influence}

Fashion refers to the prevailing atmosphere and hobbies in a certain class of society or a certain group in a certain period of time, and its members can achieve physical and mental satisfaction through the pursuit of something. Fashion consumption, as a social psychological phenomenon, is a major commercial element driving consumption and is the most vital and emotionally involved form of consumption in mass consumption which can highlight the commercial value of the market. Therefore, fashion is usually the one that can quickly attract the attention of consumers, form the upsurge and manufacturing atmosphere, convey the information in a high profile and quickly, and easily create brand products as explosive models. Moreover, when a brand has a stylish label, what it can bring to consumers is no longer a pure use value, but a manifestation of taste and personality. Can an old-fashioned toffee also trigger new fashion? How high can its added value be? Can it make consumers rush? "White Rabbit" has been integrating fashion elements in packaging, products and other aspects since 2012 in order to achieve the upgrade of brand image and expand brand influence. Among them, the packaging is particularly prominent. The packaging of "White Rabbit" is getting more and more fashionable, but the taste is still the feeling of eating as a child, and the rice paper on the sugar has not changed. It can be seen that in the process of change, the White Rabbit still pays attention to the retention of traditional elements, it tries to match the new fashion elements with the best posture.

The 200g new "giant" White Rabbit Toffee, launched in 2012, sold 2,000 "giant" White Rabbits in Shanghai's No. 1 Food Store for two weeks. In addition to the "giant" white rabbit toffee, it also introduced nostalgic iron boxed white rabbit toffee and bottled white rabbit toffee. After initially achieving certain effects with the help of fashion elements, "White Rabbit" became more confident and bold in cross-border fashion elements. The Agnes b. Brand was founded by French simple master Agnes Trouble in 1973. It is the most popular French fashion brand in Hong Kong and Japan which is known for its simplicity, uniqueness, subtlety, and understated French literary style. Its business extends to light luxury brands such as cinemas, restaurants, coffee, and art galleries. In 2014, Agnes b stated that it hopes to cooperate with some well-known old brands in the country to use new designs to provide some fashion styles for old brands, and hopes to make products look younger through design. Agnes b. has a simple design and pays attention to 
tailoring sought after by many young people. The design of the cross-border products was carried out by Agnes b.'S Greater China headquarters design team in Hong Kong, and the design was completed after one year. The overall product is still Agnes b.'s unique simple and stylish design style, which is unique in logo creativity and color application. In 2015, "Double Eleven", "White Rabbit" and Agnes b. Cross-border cooperation in a new package of White Rabbit toffee was born. "White Rabbit" and Agnes b. Come from different countries, have different brand cultures, and are in different fields. The two hold hands across borders and have a full sense of topic. The product uses the 2016 popular color released by Pantone as the packaging color. The low-saturation ice cream color of Gyeonggi blue and pink crystal is full of sweetness. The classic rabbit image of the White Rabbit and the five-pointed star of the classic elements of the Agnes b. brand are integrated into the lines and select iron materials as gift box packaging. The whole appearance is simple and full of design and fashion.

Since the product went on the market, retailers and agents from various channels have been optimistic about this cross-border cooperation product of White Rabbit and French Agnes b. Retailers such as Jiuguang Department Store, Huanmao Trade Center, and IFC Center have taken the initiative to provide venues for offline product display and personnel promotion. The e-commerce platform is also active in sales, and stock outs occurred early in the listing period. China's famous social media Douban.com, Xinhua Fashion.com, Sohu.com and other media editors praised this cross-border cooperation article. The feedback from consumers is even more acclaimed, and they have expressed their love for the new packaging with a very fashionable feel, and believe that the cooperation with Agnes b. Can make the "White Rabbit" closer to the brand characteristics of young and fashionable.

Fashion is arbitrary, transformative and cyclic. "White Rabbit" also understands this truth, and the cross-border fashion elements also keep pace with the times, and go further in the original achievements. In 2017, "White Rabbit" joined hands with "Hong Kong Pacific Coffee" to launch three stylish beverages, "White Rabbit Latte", "White Rabbit Milk Qile Bing" and "White Rabbit Coffee Qile Bing", wonderfully blended into childhood familiarity. Pacific Coffee was born in Hong Kong in 1992. In 2011, Pacific Coffee officially entered the Chinese mainland market, bringing a comfortable, warm and stylish avant-garde consumer experience to white-collar workers with good coffee, good service and a good environment. In the "Thought for the Day" updated daily by Pacific Coffee, such a famous quote has been included — Move fast and break things. Unless you are breaking stuff, you are not moving fast enough, it can be seen that it upholds subversion and leads the new Fashion concept. Since entering the Mainland market, Pacific Coffee, which advocates the fusion of Chinese and Western cultures and has unlimited creativity, has been loved and respected by many white-collar workers. The 25th anniversary of the founding of "Pacific Coffee" in 2017, the theme activity of "Honoring Classics and Reminiscing about Childhood" was launched in Hong Kong and the Mainland, which is in line with the "White Rabbit" communication concept. Moreover, the "White Rabbit" cross-border "Pacific Coffee" is different from the simple brand's cross-border approach to launch a toffee gift box. It uses the "White Rabbit" as an independent "IP" to carry out in-depth cooperation at the brand level, which is a further bold attempt to cross the "White Rabbit". Leveraging the brand influence of "Pacific Coffee" in the country chain and its extensive white-collar consumer group, Let the brand image of "White Rabbit" be younger and develop new consumer groups. "White Rabbit" can be said to be a brand with large capacity, which can achieve cross-border cooperation with multiple brands at the same time, and can reach cross-border cooperation with some unexpected objects. During the Shanghai Fashion Week in October 2017, the "White Rabbit" once again became a fashion trend which was in collaboration with the domestic cutting-edge footwear brand laber three to launch 18 large white rabbit toffee series shoes mainly in light pink, creamy white, light blue and other candy shades, highlighting the elegance, cuteness, and innocence of girls. The laber three women's shoes brand takes fun and creative lines, expressing independent women's self-awareness with unique and interesting language. Each shoe presents a different fashion flavor in a subtle and simple design. Besides its use value, it is also a fashion proverb, a manifestation of agile, lively, free and comfortable life, and a carrier of self-expression. This cross-border cooperation has achieved the perfect combination of fashionable shoes and childhood classics, so that the teenage consumer groups pursuing fashion, focusing on consumer experience, and emotional independence can feel the brand concept of White Rabbit and experience the extension of White Rabbit's products. This is also a successful practice of the complementary strategy of the "White Rabbit" cross-border consumer group. The audiences of the two brands do not overlap much, and both sides have acquired new consumers.

Cross-border marketing communications can enhance effective contact with consumers through multiple channels, successfully enhance the brand image, and then advocate new fashion trends across the country. "White Rabbit" always keeps an eye on the latest fashion trend elements, and will use the elements to cross-border marketing and communication to the end. In this process, the brand was injected with new elements and connotations a little bit, and was given more value, until a brand-new "brand network" appeared in the minds of consumers, and it did not leave the "White Rabbit" itself but enriched its brand image. 


\subsection{Brand Cross-Border Art: Enhance Brand Added Value}

As early as the mid-19th century in Europe, there was a connection between brand and art. At the beginning of the 20th century, the French brand Chanel tried to cooperate with Cubist painters. With the change of consumption concept, people's demand for spiritual and cultural consumption has increased. The art consumption market has been developed and is full of opportunities, providing full possibilities for cross-border cooperation between brands and art. When the product is integrated with art, which can meet the deeper spiritual needs of consumers, it will break through its own use value and greatly increase its added value. Today, personalized consumption is highly respected. Brands and art brands try artistic expression, which can not only give the product an artistic atmosphere, but also allow the brand to shape its cultural, artistic and traditional image in the process of communication and marketing. This is the need of the market in the era of innovation, as well as the need for personalized development of the brand.

Since the cooperation between brand and art can bring innovation and uniqueness to the brand in the era of personalized consumption, as a time-honored domestic brand in transition, "White Rabbit" also tries to grasp more opportunities brought by cross-border cooperation with art. The most typical case is the artistic cross-border cooperation between the "White Rabbit" and the cultural relics of the National Museum of China. For a long time, the museum is a place with a heavy history and solemn oldness in people's minds. It displays various cultural relics and artworks, and it seems difficult to attract young people. But in fact, it seems that a new style has become popular in recent years-"Museum +" cross-border. The State Council has proposed that state-owned cultural relic units should actively explore a reasonable income distribution mechanism and attract social forces to participate in the research and development, production and operation of cultural and creative products. As a result, a number of well-known domestic museums, led by the Palace Museum, have sought cross-border partners to achieve the integration of cultural and artistic resources and industrial resources.

The cross-border expert "White Rabbit" has undoubtedly attracted the attention of well-known museums in China. At the end of 2016, "White Rabbit" cooperated with the National Museum of China to produce cross-border cultural and creative gift boxes inspired by "Square Zun with Four Sheep", which subtly blended the two elements of "Big Rabbit" and "Square Zun with Four Sheep. "White Rabbit" has always been sweet and happy, and "Sheep" has been a symbol of meekness and auspiciousness since ancient times. Both have the same spiritual heritage, and both have unique historical heritage. The "White Rabbit-Guobo" Collector's Edition gift box perfectly blends the traditional art treasures of China with the old Chinese toffee. In today's society, it has become fashionable for brands to try to inject artistic elements and promote cross-border cultural and creative products. This model can enable brands to build high-end temperament in the process of communication marketing, and enrich the brand's cultural heritage. The "rabbit and sheep" combination has been able to converge and innovate across the millennium, which can enhance the added value of the brand and extend the brand life cycle.

\subsection{Brand Cross-Border Entertainment: Enhancing Brand Freshness}

In the social and cultural context of contemporary China, popular culture is dominating the general public's aesthetic taste with unprecedented entertainment momentum. The attraction of capital, the promotion of the media, and the burden of daily life have gradually promoted the public's aesthetic character to approach to popular culture unconsciously which create a pleasant and entertaining atmosphere. Entertainment is not a waste of time, but the inevitable demand of citizens for relaxation. Especially in today's rapid economic development, people who meet the needs of food and clothing have become increasingly personalized and advanced, and various entertainment contents and forms are welcomed by the masses. Brand cross-border entertainment makes it easier for brands to use the entertainment element to attract consumers 'attention and stimulate consumer interest and fun, which can make information transmission more efficient.

When consumers are in a happy and relaxed condition, they can more effectively deepen brand memory and increase their favorability for the brand. The form of mass entertainment has also become more and more diverse with the development of the economy, and games can be described as the new darling of entertainment recently . In the second half of 2017, "White Rabbit" joined hands with Giant Network. Guanshengyuan designed and launched the "White Rabbit-Ball Ball Combat" commemorative edition toffee packaging. "White Rabbit" teamed up with the ball game to launch a new toffee printed with the Bora Justice League package, and also launched the ball treasure exclusive commemorative version of the white rabbit toffee skin in the ball game. In terms of communication channels, "White Rabbit" used offline game activities, Internet, and posters to attack the advertising market, so that young consumers could fully experience the "White Rabbit" 's young and interesting brand image and connotation. "Ball White Rabbit" appeared at the Shanghai Station during the Ball Bao Club event. Each Shanghai Ball Bao was given a large bag of the same toffee and took a photo with the cute White Rabbit background board. In the game, the Polaroid family customized a special keyword skin for the image of the White Rabbit. As long as the nickname is changed to the keyword containing "White Rabbit toffee" before each game, it can be customized during the game. Keyword skin for White Rabbit Toffee. "White Rabbit" and Ball Ball Battle have overlapping consumer groups-teenagers who love games 
and sweets, who are willing to buy the same toffee for the surroundings of the game and who are also happy to complete tasks in the game for the new toffee skin. All supporting marketing creative stores start from the minds and acceptance habits of young consumers, cater to their interests, and guide them to reach consumption. By cross-border to make the brand more in line with consumer spending habits, thereby enhancing brand freshness.

POP-UP STORE, also known as "flash store", is a highly entertaining marketing model that can create powerful content in the short term and build customer-brand interactions. It also occupy the third position within high-frequency vocabulary that appears among young people and has grabbed the popular $\mathrm{C}$ position of the new retail business model. The content must have several characteristics such as freshness, topicality, personalization, and fun. "White Rabbit" boldly tried the latest marketing form and grasped the entertainment elements of the "flash store". "White Rabbit" and Happy Lemon cross-border cooperation, opened the 60th anniversary pop-up store, launched White Rabbit milk tea, and developed a series of peripheral products, in which consumers of different age groups can not put it down. The retro pop-up White Rabbit pop-up store has stimulated young consumers' desire to take pictures, check in, and shop. It has caused a brand sensation through personal social media communications. Consumers line up for a few hours in order to buy a cup of online red childhood memory tea. Happy Lemon is a popular beverage brand. This combination with the White Rabbit brand makes the two brands more topical, and achieves the brand 's popularity and concentrated exposure in a short time, which has brought huge traffic and Popularity.

\section{Grasp Cross-Border Marketing Issues and Formulate a Positive Future Development Strategy}

For traditional "old-time" brands, it is necessary to ensure the quality of cross-border products and improve product practicality. Brand cross-borders can't cross borders for cross-borders. They can't just consider whether the product can drive traffic or cater to consumers. Instead, they must integrate thinking, consider cross-border objects, time nodes, and communication methods. Launches practical, high-quality, cross-border products with bursting points based on consumer demand, thereby seizing new market opportunities and innovating brand image.

In the initial stage of choosing a cooperative brand, it is necessary to be cautious to decide if traditional brand cross border. Before choosing a cooperating brand. it should be considered whether the brand concepts of the two parties are in line with each other, and whether the consumer groups have overlapped. Avoid cooperation with brands that are inconsistent with the brand concept, so as not to destroy the existing image, cause consumer dislike, and blur the brand positioning. Choosing the right partner is convenient for resource platform sharing and targeted promotion of products, so as to achieve efficient cross-border cooperation.

Based on the existing cross-border cases, companies and brands should also update cross-border marketing models at any time to attract more loyal consumers. For "White Rabbit", more and more fresh communication methods can be tried to ignite cross-border activities, such as live delivery, physical store creation, seasonal change of themes, etc., and continue to bring consumers freshness. Learn lessons from cross-border cases to make cross-border decisions that are more in line with the "White Rabbit" brand demands and more suitable for the "White Rabbit" transformation.

For brands, cross-borders can't just float on the surface and only pursue temporary topical levels and traffic, especially traditional veteran brands. They should also dig deeper into the cultural integration between brands to achieve long-term development. Behind the 60-year-old "White Rabbit" is the fruit of the brand's painstaking efforts. It is the times and the market that compels the young to embark on the road of youth. "Rabbit" is steadily playing a leading role in the toffee industry for a long time and continues to take root in the hearts of new "generation after generation" consumers.

\section{Summary}

Cross-border marketing can form a win-win situation for both parties and establish a new brand image in this process by seeking integration between industries, finding clever junctions between brands, and working together to create products that appeal to consumers. In recent years, while attracting young consumers, we should pay attention to maintaining product quality and the reputation of established brands with cross-border marketing of established brands taking up endlessly. In terms of cross-border forms, attention should be paid to innovative content forms to avoid consumer resentment caused by homogeneity and tackiness.

The author believes that cross-border is only a means to achieve brand rejuvenation for traditional brands, which can effectively attract young consumers' attention for a short time. However, this marketing model can only produce phenomenal-level marketing effects. It is difficult for online celebrity products to maintain a long-term popularity. Once the heat is low, they will fall into an embarrassing situation. For the time-honored brand that has been passed down from generation to generation, a truly reasonable cross-border approach is to always pay attention to the correct image in the fierce market competition, carry out long-term, regular and regular cross-border marketing, and use creativity as the guide for product development. , Carry out marketing strategy planning suitable for the brand. Only in this way can we attract the attention of young consumer groups for a long time, penetrate the younger generation without causing them to get bored and realize the rejuvenation of the old brand. 


\section{References}

Hong, T., \& Tong, H. M. (2018). China's Fashion Consumption in the New Era of Consumption Upgrade [J].Consumer Economy, 34(02), 28-36.

Hou, Y. (2018). Old national brands also play "cross-border" [J]. China Economic Weekly, 2018(41), 84-86.

Li, L. L. (2019). Feasibility Study on the Cross-border Cooperation of "National Toffee" White Rabbit Brand [J]. Chinese Art, 2019(05), 18-25.

Li, N., \& Chen, Y. P. (2019). Opening the brain hole Cross-border marketing [J]. Enterprise Management, 2019(10).

Ni, W. R. (2010). Cross-border research on brand marketing communication [D]. Suzhou University, 2010.

Wang, S. T. (2016). Research on the Cross-Border Cooperation between Brand and Art [D]. Donghua University, 2016.

Wu, Z. Y. (2018). "White Rabbit" and Cross-border Marketing [J]. Shanghai Packaging, 2018(04), 6-8.

Yang, Y. (2018). Research on Story Communication and Traditional Brand Image Remodeling [D]. East China Normal University, 2018.

Zang, N. (2012). Research on Contemporary Literary Entertainment [D]. Liaoning University, 2012.

\section{Copyrights}

Copyright for this article is retained by the author(s), with first publication rights granted to the journal.

This is an open-access article distributed under the terms and conditions of the Creative Commons Attribution license which permits unrestricted use, distribution, and reproduction in any medium, provided the original work is properly cited. 